\title{
Optimal Pre-Pregnancy Body Mass Index Cut-Offs for Obesity in Japan
}

\author{
Shunji Suzuki
}

\section{To the Editor}

Based on our earlier studies in Japan [1, 2], we understand that we need to be more tolerant for the gestational weight gain in Japanese woman than ever, especially in overweight women. However, we have not well examined the influence of the maternal pre-pregnancy physique on the perinatal outcomes in the overweight and/or obesity women in Japan.

To date, some pre-pregnancy body mass indexes (BMIs; $\mathrm{kg} / \mathrm{m}^{2}$ ) cut-offs have been examined to predict the high risk BMI levels as obesity associated with the adverse perinatal outcomes [3-5]. For example, in Japan, the Japanese Ministry of Health, Labour and Welfare (JMHLW) guideline has defined pre-pregnancy BMI of $\geq 25$ as obesity [3], while the Institute of Medicine (IOM) guideline in the USA has defined pre-pregnancy BMI of $\geq 30$ as obesity associated with the increased risk of both neonatal macrosomia and cesarean delivery [4]. In 2004, in addition, the World Health Organization (WHO) recommended the classification of pre-pregnancy BMI of $\geq 27.5$ as obesity in Asian populations [5]. To (re-)assess the optimal pre-pregnancy BMI cut-offs for obesity in Japanese women associated with the perinatal outcomes, we examined the perinatal outcomes in Japanese singleton pregnancy of prepregnancy BMI 25.0 - 27.4, 27.5 - 29.9 and $\geq 30.0$ compared with that of BMI 18.5 - 24.9 as control.

The protocol for this study was approved by the Ethics Committee of the Japanese Red Cross Katsushika Maternity Hospital. Informed consent concerning analysis from a retrospective database was obtained from all subjects.

We reviewed the obstetric records of singleton pregnant Japanese women who delivered at our institute at $\geq 22$ weeks' gestation from April 2012 through November 2016. Data were expressed as mean \pm standard deviation or number (percentages). Cases and controls were compared by means of Student's $t$-test for continuous variables, and the $\mathrm{X}^{2}$ or Fisher's exact test for categorical variables. Differences with $\mathrm{P}<0.05$ were

Manuscript accepted for publication December 21, 2016

Department of Obstetrics and Gynecology, Japanese Red Cross Katsushika Maternity Hospital, 5-11-12 Tateishi, Katsushika-ku, Tokyo 124-0012, Japan. Email: czg83542@mopera.ne.jp

doi: https://doi.org/10.14740/jocmr2883w considered significant.

Table 1 shows the clinical description and perinatal outcomes in Japanese singleton pregnancies based on the maternal pre-pregnancy BMI cut-offs for obesity according to the JMHLW, WHO Asian and IOM BMI guidelines. There were no significant differences in the clinical description such as maternal age, parity or maternal height among the four groups. To predict the incidence of maternal impaired glucose tolerance in Japanese pregnant women, the Japanese (JMHLW BMI) cut-off seemed to better than the other two cut-offs; however, to predict the incidence of other complications such as maternal hypertensive disorders, cesarean delivery and neonatal macrosomia, the other cut-offs seemed to be better than the Japanese cut-off. Based on the current observation, we may be also required to be more tolerant for the maternal pre-pregnancy physique than ever, especially in overweight women of BMI 25 - 27.4. To confirm these, a larger study is needed.

\section{References}

1. Suzuki S. Optimal Weight Gain During Pregnancy in Japanese Women. J Clin Med Res. 2016;8(11):787-792.

2. Suzuki S. Gestational Weight Gain in Japanese Women With Favorable Perinatal Outcomes. J Clin Med Res. 2017;9(1):64-66.

3. Rasmussen KM, Yaktine AL, Eds. Weight gain during pregnancy: reexamining the guidelines. Washington, DC: National Academies Press; 2009.

4. Promotion Council for Healthy Parents and Children 21 (second edition) (in Japanese). Ministry of Health, Labour and Welfare, 2015. http://rhino3.med.yamanashi. ac.jp/sukoyaka2/english.html (December 13, 2016).

5. Appropriate body-mass index for Asian populations and its implications for policy and intervention strategies. Lancet. 2004;363(9403):157-163. 
Table 1. Clinical Description and Perinatal Outcomes in Japanese Singleton Pregnancies Delivered at $\geq 22$ Weeks' Gestation Based on the Maternal Pre-Pregnancy BMI Cut-Offs for Obesity According to the JMHLW, the WHO Asian and the IOM BMI Guidelines

\begin{tabular}{|c|c|c|c|c|}
\hline Pre-pregnancy BMI & Control & Obesity of JMHLW & Obesity of WHO & Obesity of IOM \\
\hline BMI & $18.5-24.9$ & $25.0-27.4$ & $27.5-29.9$ & $\geq 30.0$ \\
\hline Number & 3,785 & 225 & 151 & 110 \\
\hline Average & $21.1 \pm 1.5$ & $26.4 \pm 0.6$ & $28.7 \pm 0.8$ & $33.0 \pm 2.4$ \\
\hline Maternal height (cm) & $159 \pm 5.3$ & $157 \pm 5.9$ & $158 \pm 5.0$ & $158 \pm 6.6$ \\
\hline Body weight at pre-pregnancy (kg) & $52.9 \pm 5.1$ & $65.3 \pm 5.2 *$ & $72.5 \pm 5.0^{*}$ & $82.5 \pm 8.5^{*}$ \\
\hline Body weight at delivery (kg) & $63.5 \pm 6.5$ & $756 \pm 7.7^{*}$ & $80.8 \pm 7.1^{*}$ & $87.6 \pm 9.2^{*}$ \\
\hline Gestational weight gain (kg) & $10.3 \pm 5.7$ & $10.3 \pm 4.7$ & $7.1 \pm 5.6$ & $5.0 \pm 5.0^{*}$ \\
\hline Maternal age (years) & $32.7 \pm 5.6$ & $32.7 \pm 6.0$ & $32.7 \pm 6.0$ & $31.9 \pm 5.0$ \\
\hline$<20$ years & $80(2.1 \%)$ & $4(1.2 \%)$ & $2(1.3 \%)$ & $0(0 \%)$ \\
\hline$\geq 35$ years & $1,338(35.3 \%)$ & $75(23.1 \%)$ & $57(31.7 \%)$ & $29(26.4 \%)$ \\
\hline Nulliparity & $1,838(48.0 \%)$ & $96(43.7 \%)$ & $61(40.4 \%)$ & $57(51.8 \%)$ \\
\hline Hypertensive disorders & $310(8.2 \%)$ & $25(11.1 \%)$ & $23(15.2 \%)^{*}$ & $25(22.7 \%)^{*}$ \\
\hline Impaired glucose tolerance & $82(2.2 \%)$ & $12(5.3 \%)^{*}$ & $11(7.3 \%)^{*}$ & $17(15.5 \%)^{*}$ \\
\hline Preterm delivery & $249(8.6 \%)$ & $14(6.2 \%)$ & $8(5.3 \%)$ & $6(6.5 \%)$ \\
\hline Cesarean delivery & $538(14.2 \%)$ & $35(15.6 \%)$ & $18(11.9 \%)$ & $36(32.7 \%)^{*}$ \\
\hline Neonatal birth weight (g) & $2,990 \pm 441$ & $3,081 \pm 446$ & $3,148 \pm 411^{*}$ & $3,246 \pm 399 *$ \\
\hline$<2,500 \mathrm{~g}$ & $399(10.5 \%)$ & $18(8.0 \%)$ & $6(4.0 \%)^{*}$ & $2(1.3 \%)^{*}$ \\
\hline$\geq 4,000 \mathrm{~g}$ & $28(0.7 \%)$ & $4(1.8 \%)$ & $6(4.0 \%)^{*}$ & $5(6.5 \%)^{*}$ \\
\hline Postpartum hemorrhage $\geq 1,000 \mathrm{~mL}$ & $356(9.4 \%)$ & $28(12.4 \%)$ & $21(13.9 \%)$ & $15(9.9 \%)$ \\
\hline
\end{tabular}

Data are expressed as mean \pm standard deviation or number (percentages). ${ }^{*} \mathrm{P}<0.01$ vs. control. BMI: body mass index; JMHLW: Japanese Ministry of Health, Labour and Welfare; WHO: World Health Organization; IOM: the Institute of Medicine. 